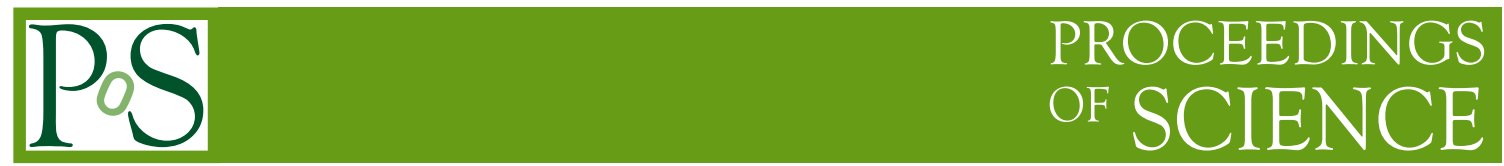

\title{
CMS Results on Searches for Physics beyond the Standard Model
}

\section{Wolfgang WALTENBERGER ${ }^{* \dagger}$}

HEPHY Vienna

E-mail: walten@cern.ch

To this date CMS has performed a wide range of searches for physics beyond the standard model. The program comprises both broad, inclusive searches as well as exclusive, specialised approaches. In this article, recent results with proton-proton collision data both at 7 and $8 \mathrm{TeV}$ are summarized, with a focus on presenting some highlights of the CMS search program.

LHC on the March

20-22 November 2012

Institute for High Energy Physics, Protvino, Moscow region, Russia

\footnotetext{
${ }^{*}$ Speaker.

†n behalf of the CMS Collaboration.
} 


\section{Introduction}

The year 2012 has been exciting for the LHC experiments, the obvious highlight being the discovery of a Higgs-boson-like particle [1], [2]. If the new bosonic particle is indeed the longsought Higgs boson, the hierarchy problem arises immediately: what stabilizes the mass of the Higgs boson at $\approx 125 \mathrm{GeV}$ ? Do we live in a fine-tuned universe, or is there a yet undiscovered symmetry responsible for the low Higgs mass? Theories which avoid fine-tuning to explain a low Higgs mass are commonly referred to as "natural" theories.

The CMS collaboration pursues a wide physics program in search for such a potential new symmetry, and a natural solution to the hierarchy problem. This program if formally split up into two categories, according to the theoretical motivation behind the searches: Searches for signatures inspired by supersymmetry (SUS), and searches for non-supersymmetric, "exotic" physics beyond the standard model (EXO). The majority of all CMS publications of 2012 falls into the EXO category.

It is the aim of this document to summarize recent efforts of the CMS collaboration in these two categories. Results that are based on the full $7 \mathrm{TeV}$ dataset from 2011 are shown, as well as $8 \mathrm{TeV}$ studies with data collected in the 2012 run. The document is structured as follows. The searches for signs of supersymmetry are discussed in Sec 2 . The exotic searches will be the topic of Sec. 3. Finally, Sec. 4 summarizes the status of the BSM physics searches.

\section{Searches for supersymmetry}

Supersymmetry $[3,4,5,6,7,8]$ is one of the theoretically most promising candidates to solve several outstanding problems in particle physics, one of which being the hierarchy problem, as mentioned in the introduction. Supersymmetry introduces a new fermionic super-partner to every one of the standard model's bosonic degrees of freedom, and vice versa. The supersymmetric partner particles can the divergences of large radiative corrections to the Higgs boson mass, allowing it to remain naturally light near the electroweak scale. Supersymmetry can thus be a "natural" theory. If so-called R-parity is imposed on a supersymmetric theory, then the lightest supersymmetric particle (LSP) becomes stable, can be neutral, and is therefore a dark-matter candidate.

Already the minimal supersymmetric extension to the standard model (MSSM) counts more than 100 free parameters. This number clearly is too high to quantify the experiments' search results. Therefore different ways to reduce the number of the model's free parameters have been devised:

- Constrained models $[9,10,11]$ typically impose conditions on the model parameters in the high-energy regime. The "constrained MSSM" (cMSSM) is defined by $4+1$ free parameters.

- Phenomenological models $[12,13,14,15]$ on the other hand try to parametrize the MSSM with $18 / 19$ free parameters at the SUSY scale.

- Simplified models $[16,17,18,19]$ do not pretend to be "fundamental" theories; instead, they are intended to describe experimental results in terms of effective Lagrangiangs with only a very limited set of free parameters, typically two or three particle masses. 
Following the general trend in the experimental collaborations, the results in this document will be mostly given in terms of the third category.

The CMS SUSY group has pursued a wide range of searches for signs of supersymmetry [20, $21,22,23,24,25,26,27]$.

In what follows, the results for the search for single-leptonic stop decays [23], the search for weakino production [22], and the search for RPV SUSY [25] will be discussed in more detail.

\section{Search for single-leptonic stop decays}

The CMS collaboration's latest search for stop decays in the single-leptonic channel [23] requires a single isolated electron or muon and at least one jet that is identified as originating from a b quark. The data sample used corresponds to an integrated luminosity of $9.7 \mathrm{fb}^{-1}$ of pp collisions at a centre-of-mass energy of $8 \mathrm{TeV}$. The signal regions comprise selections based on the missing energy and on the $M_{\mathrm{T}}$ variable, where $M_{\mathrm{T}}$ is the "transverse mass" of the lepton plus missing energy:

$$
M_{\mathrm{T}}^{2}=2 p_{T}^{l} \mathrm{E}_{\mathrm{T}}(1-\cos \phi),
$$

where $p_{T}^{l}$ and $\mathrm{E}_{\mathrm{T}}$ are the magnitude of the transverse lepton and the missing transverse momentum, respectively, and $\phi$ denotes the angle between the lepton and the missing energy. The lepton is taken to be massless.

Figure 1 shows the distribution of $M_{\mathrm{T}}$ for events that have met the missing energy requirement. The region with $M_{\mathrm{T}}>150 \mathrm{GeV}$ - Figure $1(\mathrm{a})$ - and $M_{\mathrm{T}}>120 \mathrm{GeV}-$ Figure $1(\mathrm{~b})$ - correspond to two different signal regions: no excess can be seen. The null result is interpreted in the context of two different simplified models: stop-stop pair production with a direct decay to top and the LSP - shown in Figure 2(a), and stop-stop pair production with an intermediate cascade decay through charginos, Figure 2(b).

\section{Search for production of weakinos}

One CMS analysis [22] studies final states with exactly three leptons, four leptons, two samesign leptons, two opposite-sign-same-flavor leptons plus two jets, or two opposite-sign leptons inconsistent with $\mathrm{Z}$ boson decay. $9.2 \mathrm{fb}^{-1}$ of data at $8 \mathrm{TeV}$ have been considered. Different methods are employed for the different final state signatures; Figure 3(a) shows a summary plot of all results obtained in Ref. [22], weakino decays via sleptons and decays via gauge bosons are considered. Fig 3(b) zooms in on the decay modes through gauge bosons - the red combination curve in this plot corresponds to the dashed blue line in Figure 3(a): for gauge boson decays, chargino/neutralino masses only up to $300 \mathrm{GeV}$ can be excluded.

\section{Search for R-parity violating (RPV) supersymmetry}

Recently, also results for RPV supersymmetry have been made public [25]. The advantage of these searches is obviously that no cut on $\mathrm{E}_{\mathrm{T}}$ is applied. $9.2 \mathrm{fb}^{-1}$ of $8 \mathrm{TeV}$ are the base of this search; the multilepton events are categorized based on the identity and kinematics of the leptons. Figure 4 shows the interpretation in terms of the "hadronic RPV" model: the Feynman diagram, Figure 4(a), and the result, Figure 4(b). Figure 5 shows the Feynman diagram and the results for the "stop RPV" model. Ref. [25] shows re-interpretations in several additional models. Depending 


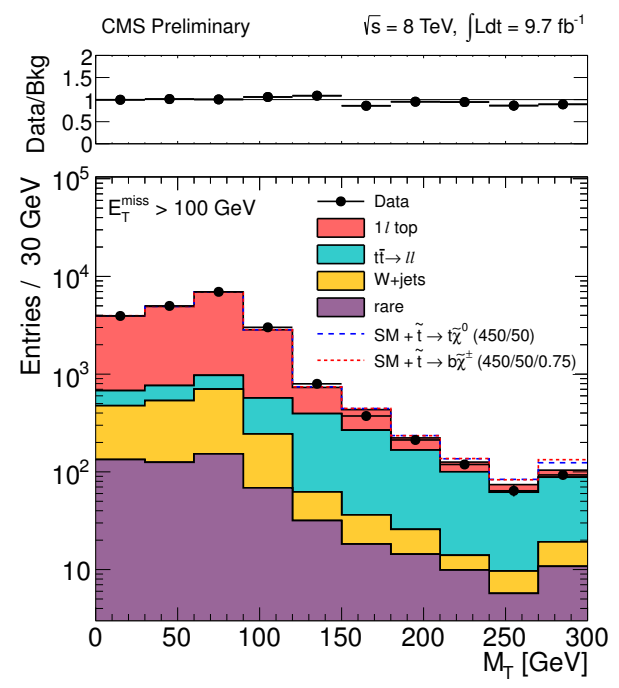

(a) $\mathrm{E}_{\mathrm{T}}>100 \mathrm{GeV}$
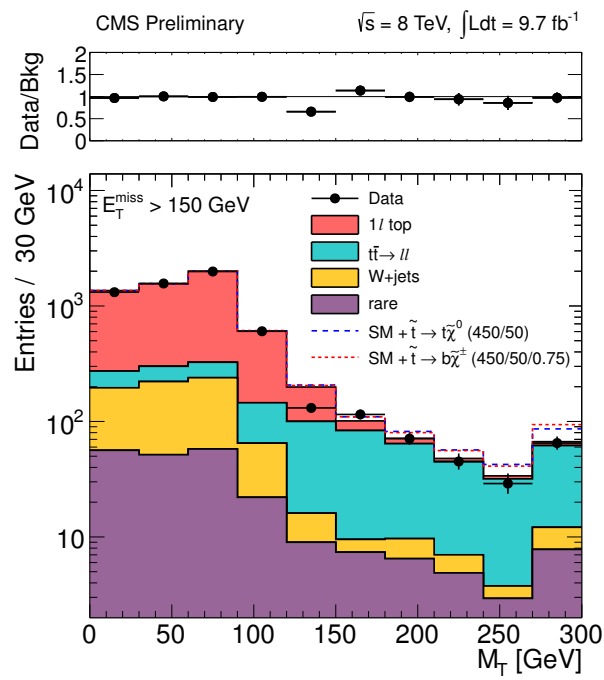

(b) $\mathrm{E}_{\mathrm{T}}>150 \mathrm{GeV}$

Figure 1: $M_{\mathrm{T}}$ distributions, for $\mathrm{Z}_{\mathrm{T}}>100 \mathrm{GeV}$ (left) and for $\mathrm{Z}_{\mathrm{T}}>150 \mathrm{GeV}$ (right), for the single-leptonic stop search [23].

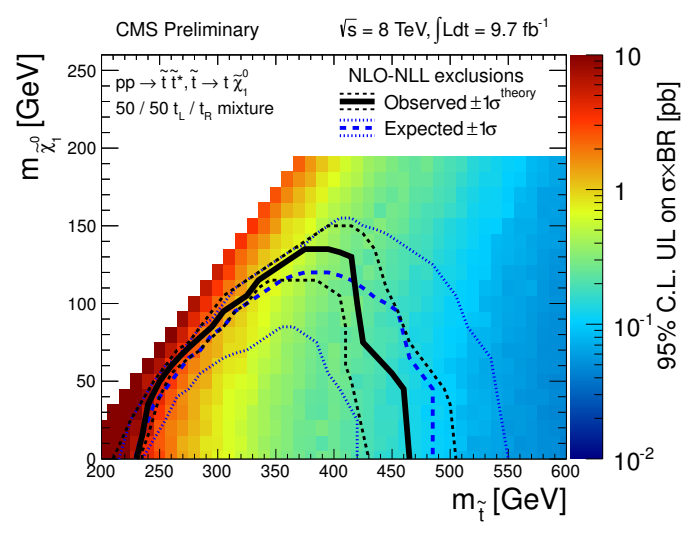

(a) $\tilde{\mathrm{t}} \rightarrow t \widetilde{\chi}_{1}^{0}$

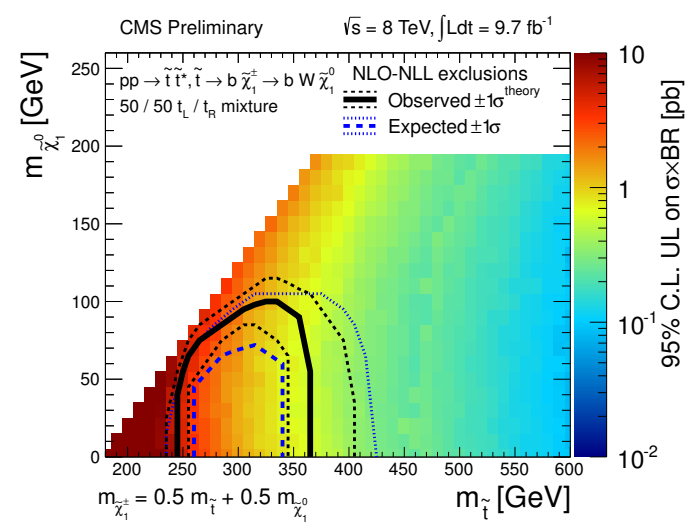

(b) $\tilde{\mathrm{t}} \rightarrow b \tilde{\chi}_{1}^{ \pm}, \tilde{\chi}_{1}^{ \pm} \rightarrow W \tilde{\chi}_{1}^{0}$

Figure 2: Single-leptonic stop decays, interpretations in SMS space, [23].

on the specific model assumptions, and for the assumption of $\mathrm{BR}=1$ one finds $95 \%$ exclusion limits for stop and sbottom quarks of up to $900 \mathrm{GeV}$, gluinos and squarks are excluded for values below $\approx 1200$ and $1700 \mathrm{GeV}$, respectively. 


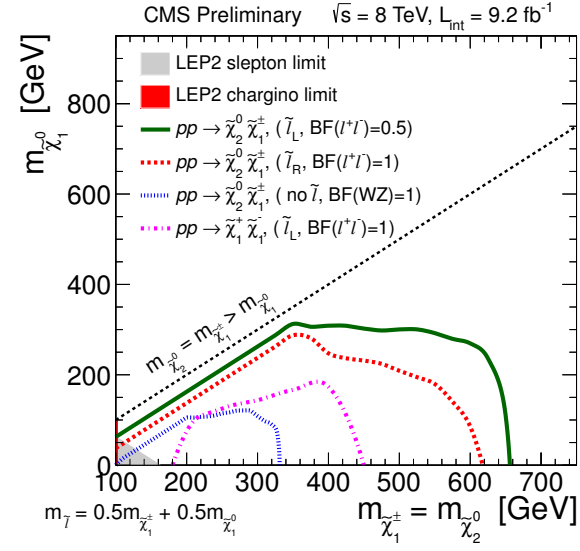

(a) Summary, weakino production

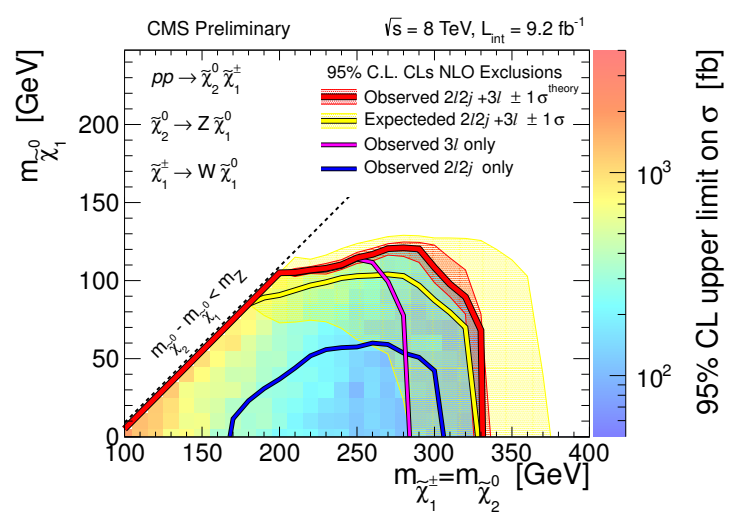

(b) Weakino production, decays through vector bosons

Figure 3: Summary plots, weakino production, [22].

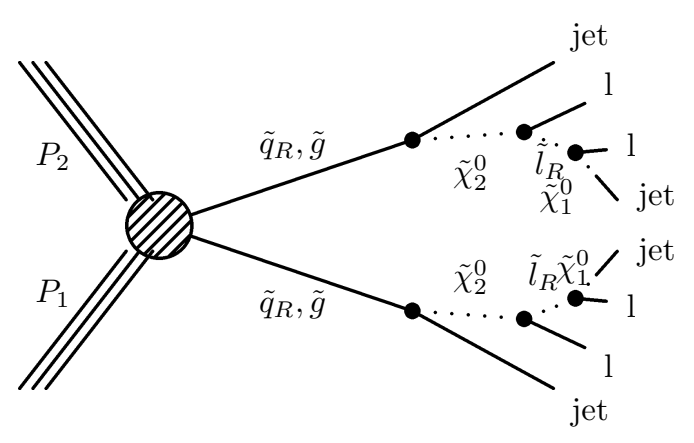

(a) Feynman diagram of the hadronic RPV model

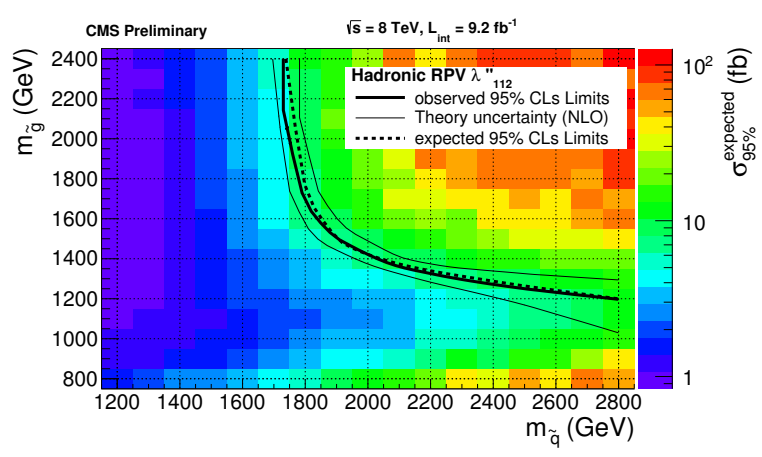

(b) Result for the hadronic RPV model

Figure 4: Feynman diagram and results for the hadronic RPV model, [25].

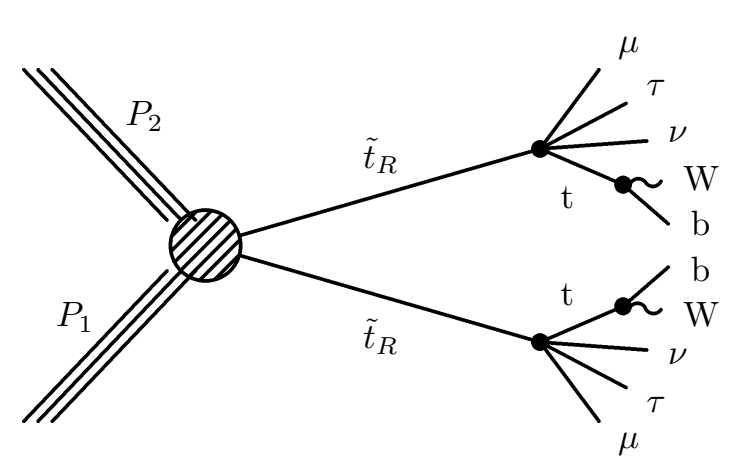

(a) Feynman diagram

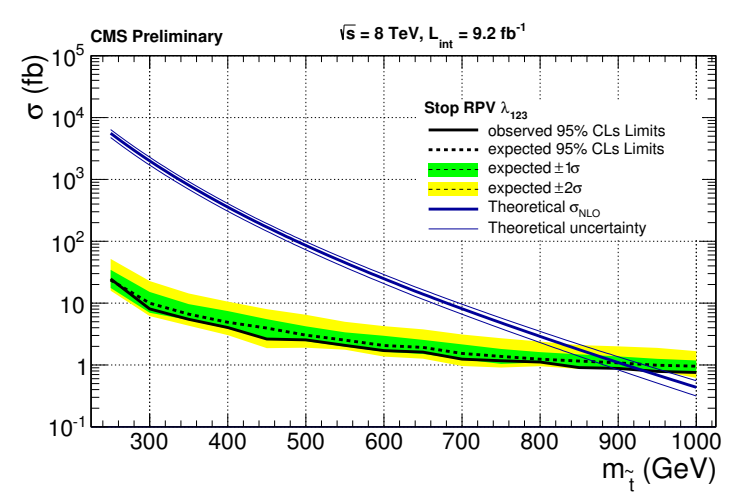

(b) Results

Figure 5: Feynman diagram and results for the stop RPV model, [25]. 


\section{Summary: searches for supersymmetry}

The overall status of the CMS searches for supersymmetry is summarized in Figure 7. The naturalness argument has since long been a driving theoretical motivation for supersymmetry at low energy scales [28]. Among other things it demands relatively light bottom and top quark partners $\left.\mathrm{m}\left(\tilde{\mathrm{t}}_{1}\right), \mathrm{m}\left(\tilde{\mathfrak{t}}_{2}\right), \mathrm{m}\left(\tilde{\mathrm{b}}_{1}\right) \lesssim 500 \mathrm{GeV}\right)([29,30,31])$. Figure 6 summarizes the experimental evidence. It must be noted however that all exclusion lines are drawn for an assumed branching ratio of 1 .

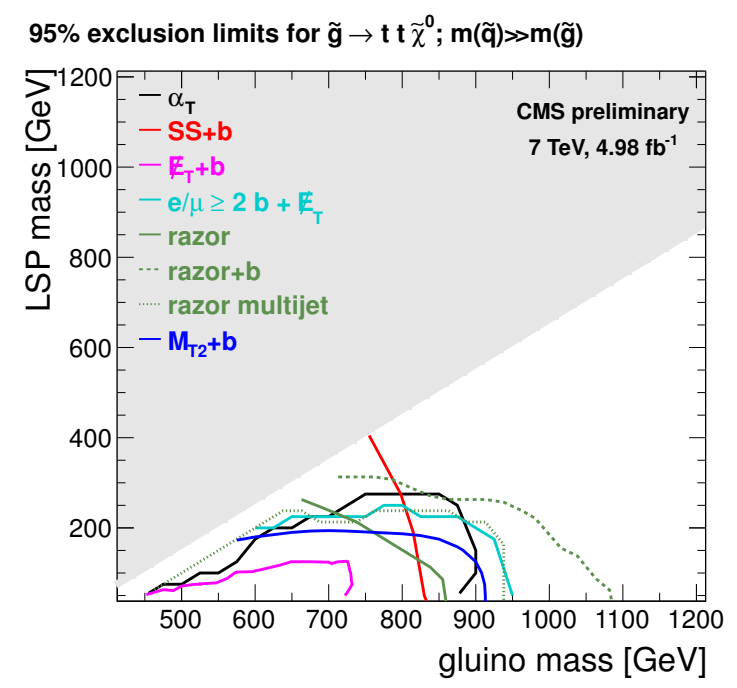

(a) $\mathrm{T} 1 \mathrm{tttt}$

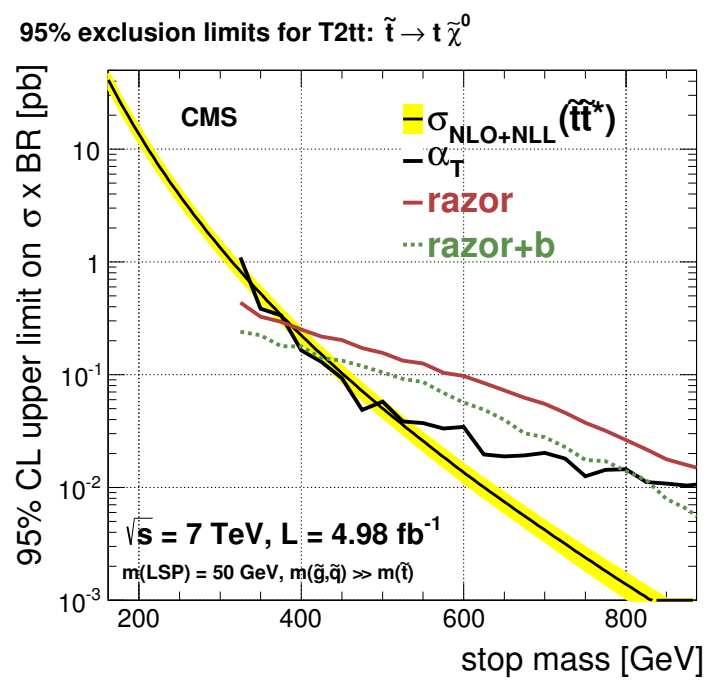

(b) $\mathrm{T} 2 \mathrm{tt}$

Figure 6: Summary plots for gluino-mediated (T1tttt) and direct (T2tt) stop production. 


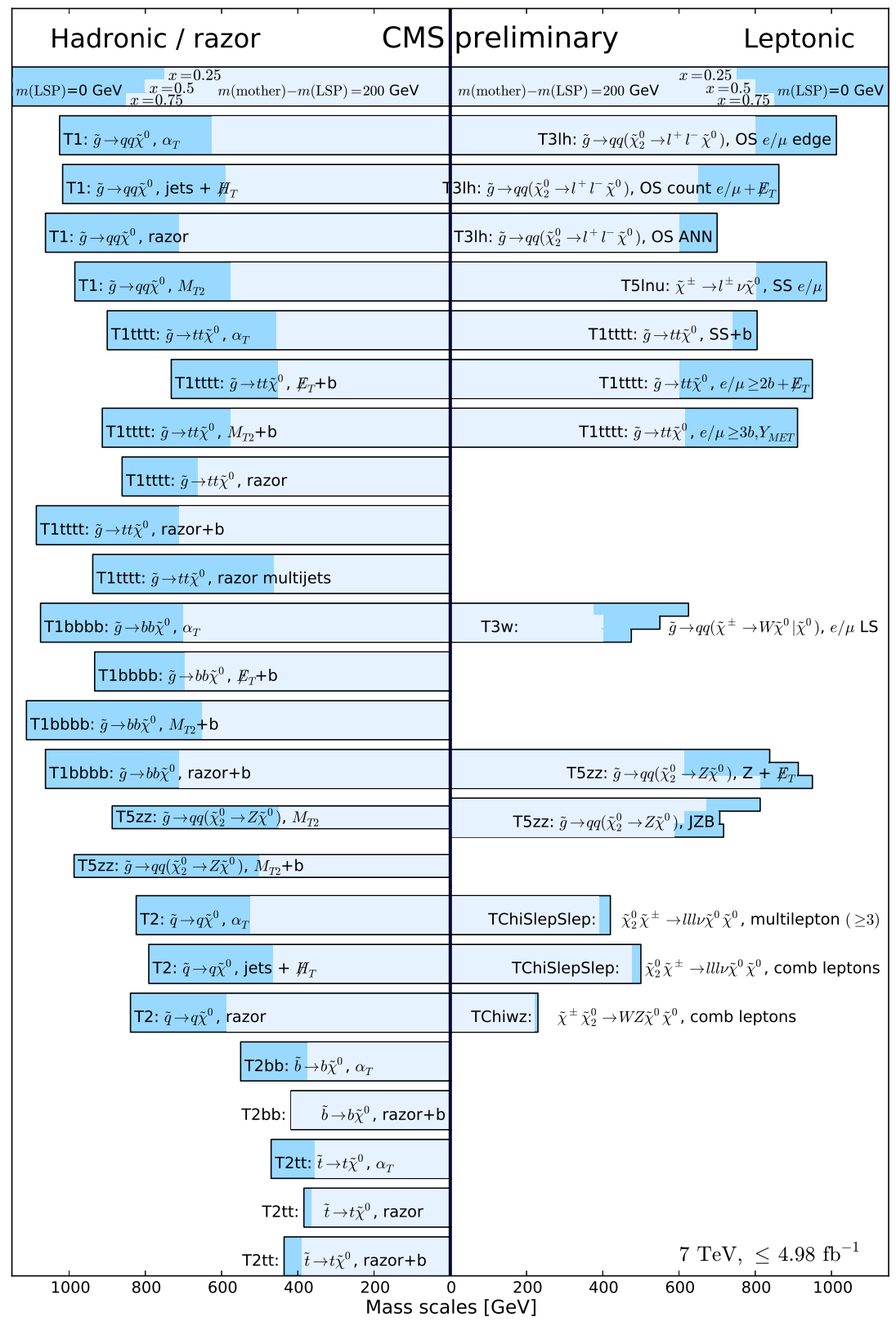

Figure 7: Summary bar plot, supersymmetry. The dark blue bars denote the limits for $\mathrm{m}(\mathrm{LSP})=0$, light blue represents lmits for $\mathrm{m}$ (mother)-m(LSP) $=200 \mathrm{GeV}$. 


\section{Exotic searches in CMS}

CMS has also made significant progress in their searches for exotic physics. In the following, examples in the searches for W' bosons, dijet resonances, and black holes will be given.

\subsection{W' bosons}

Searches for additional gauge bosons have been performed by the CMS experiment. A search for a heavy charged gauge boson (W') can be found among the results made public [32]. A potential signal would manifest itself as an enhanced production rate of events with a charged lepton and a neutrino, the $M_{\mathrm{T}}$ variable is a natural choice for a discriminating variable. Figure 8 shows the distributions of $M_{\mathrm{T}}$ in the electron and the muon channels. No excess is to be seen. Figure 9 interprets the null-results in the context of a sequential standard model (SSM) [33] W' boson and for contact interactions ("HNC" model).

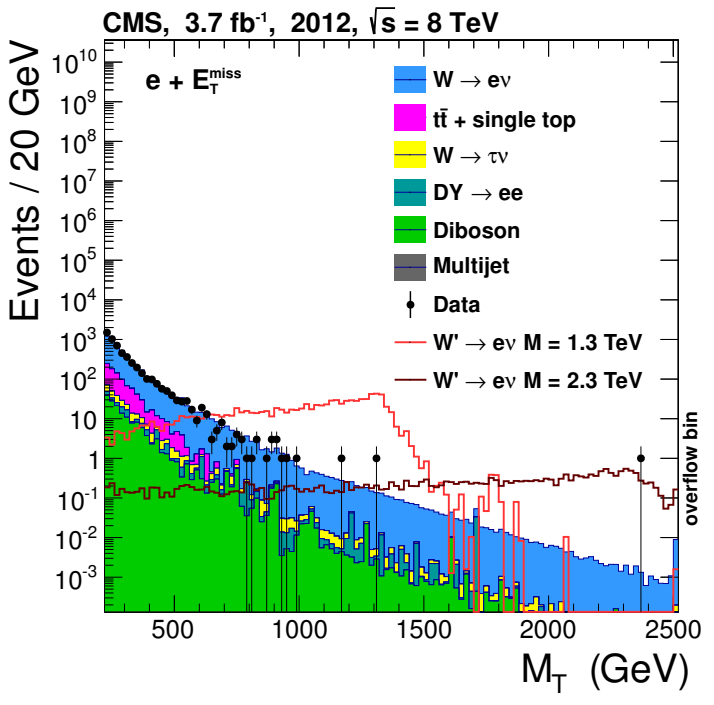

(a) $M_{\mathrm{T}}$, in the electron channel

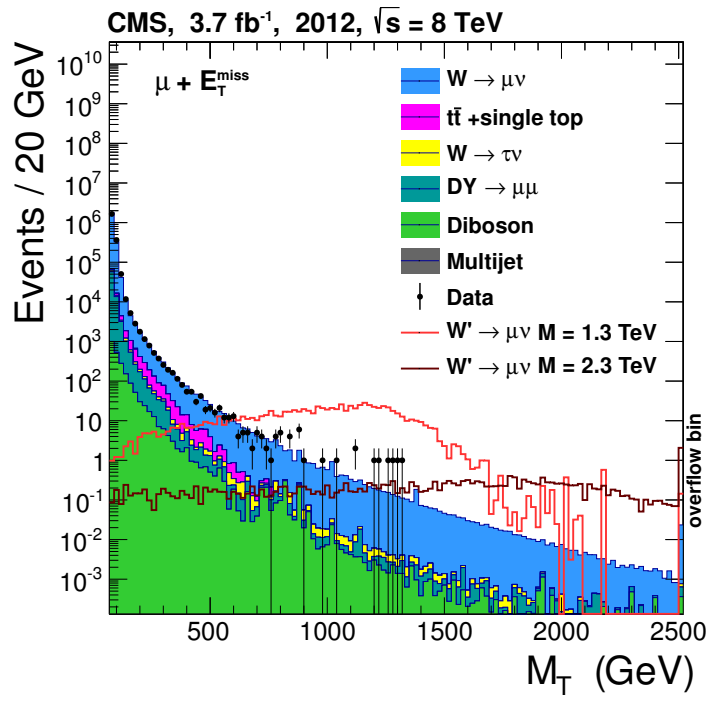

(b) $M_{\mathrm{T}}$, in the muon channel

Figure 8: $M_{\mathrm{T}}$ distributions of the $\mathrm{W}^{\prime}$ searches, [32]

\subsection{Dijet resonances}

A wide plethora of models for BSM physics predicts narrow resonances in the dijet channel ([35]. CMS reports no evidence for new narrow resonance production [36], with $4 \mathrm{fb}^{-1}$ of $8 \mathrm{TeV}$ having been analysed. Figure 10(a) shows the observed dijet mass spectrum, and gives examples for what signals might look like. Figure 10(b) gives upper limits on production cross sections (times acceptance times branching ratios), showing also the predicted cross sections for two different models.

\subsection{Black holes}

A search for microscopic black hole production in pp collisions is also reported by the CMS experiment [34]. Events of the $8 \mathrm{TeV}$ data sample corresponding to an integrated luminosity of 


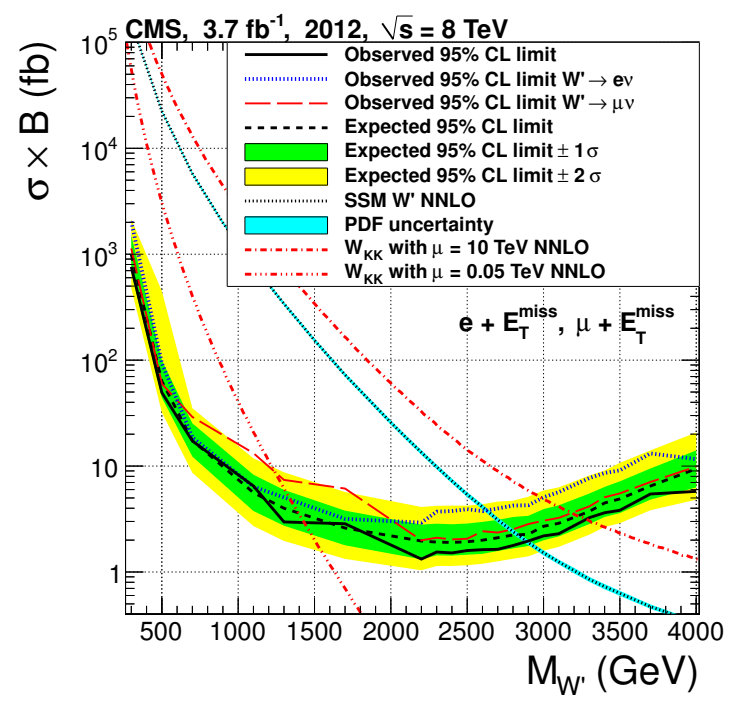

(a) SSM

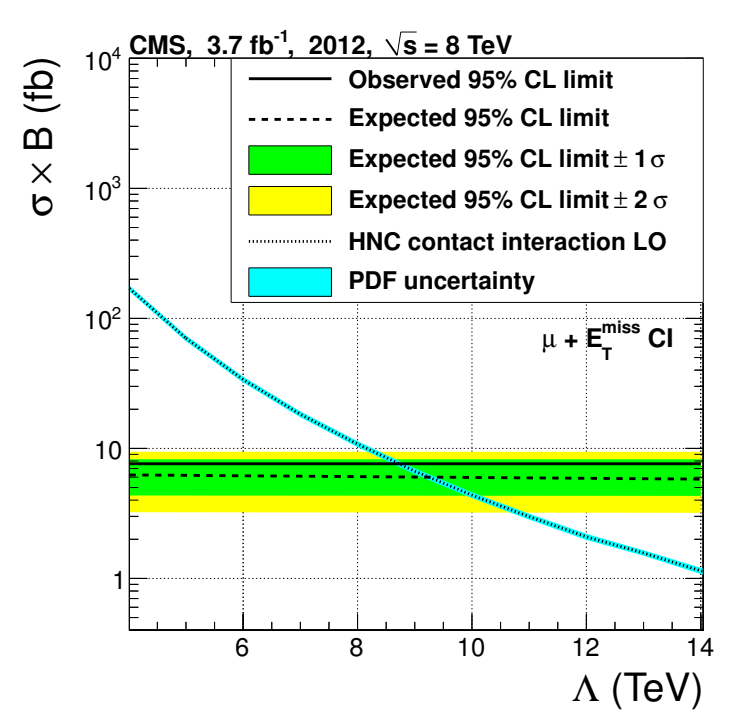

(b) contact interaction

Figure 9: Interpretations of the results of the W' search, [32]

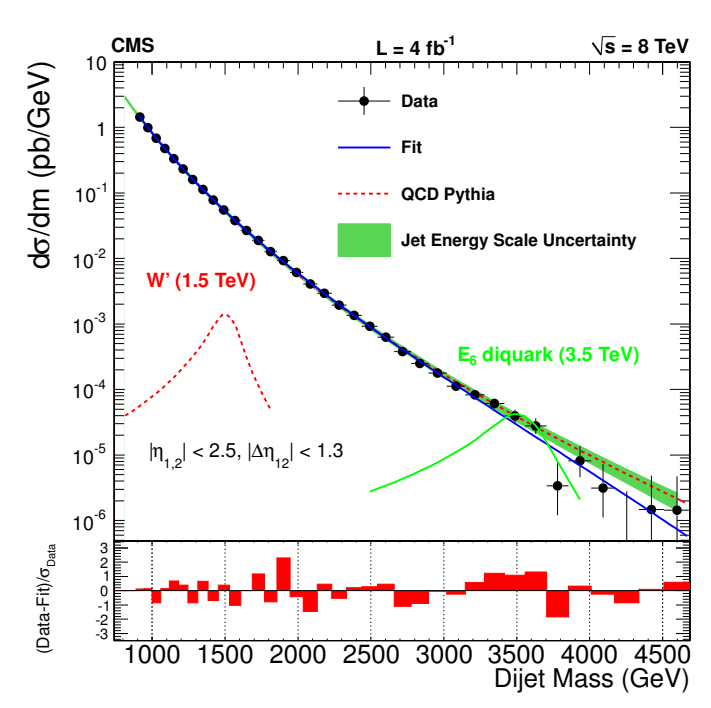

(a) dijet mass spectrum

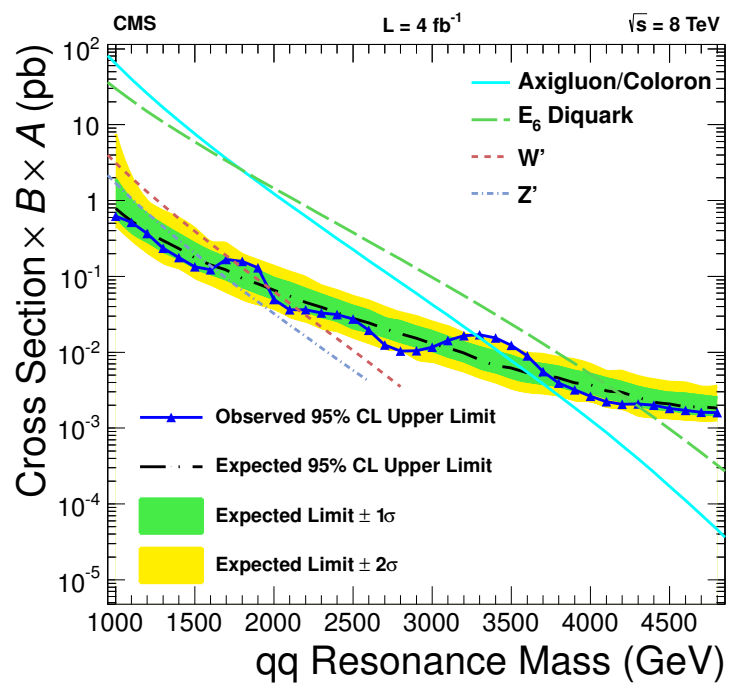

(b) observed upper limits

Figure 10: Left: The observed dijet mass spectrum (points), and fitted with a smooth curve (solid line). The predicted QCD background is shown as a dashed red line. The distribution expected for two typical signals (1.5 TeV W' boson and E6 diqaurk at 3.5 TeV) are shown. Right: 95\% CL observer upper limits on $\sigma x B x A$ are shown together with reference cross section for a few models. E.g. E6 diquarks can be excluded up to an invariant mass of $\approx 4 \mathrm{TeV},[36]$.

$3.7 \mathrm{fb}^{-1}$ have been analyzed for the presence of multiple energetic jets, leptons, and photons. Figure 11 shows a typical candidate event. The analysis sees no signs of new physics. Limits are reported in a model-independent fashion [Figure 12(a)] and specifically for semiclassical black 
holes - Figure 12(b).

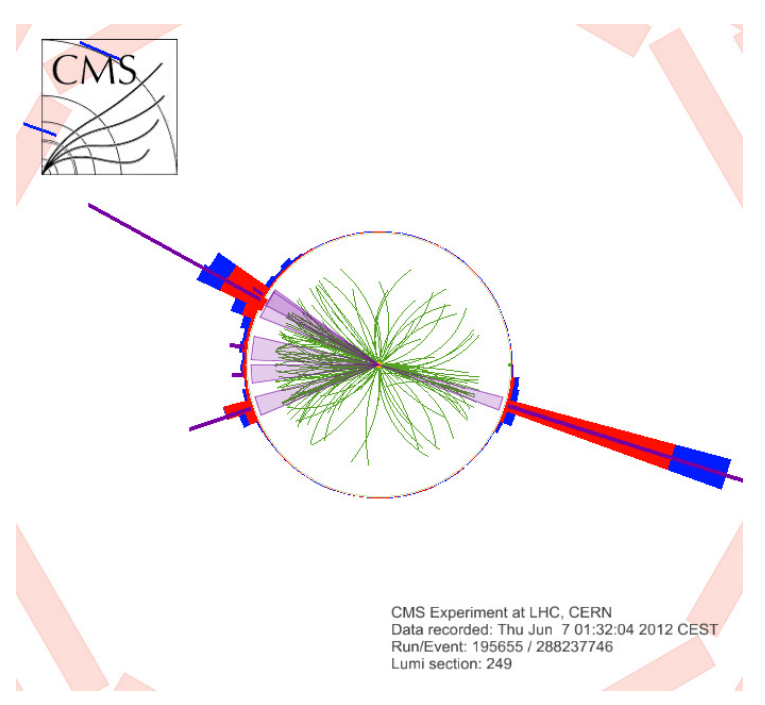

Figure 11: Rho-phi view of 6-jet (magenta cones) candidate black-hole event, $\mathrm{ST}=3.5 \mathrm{TeV}$, [34]

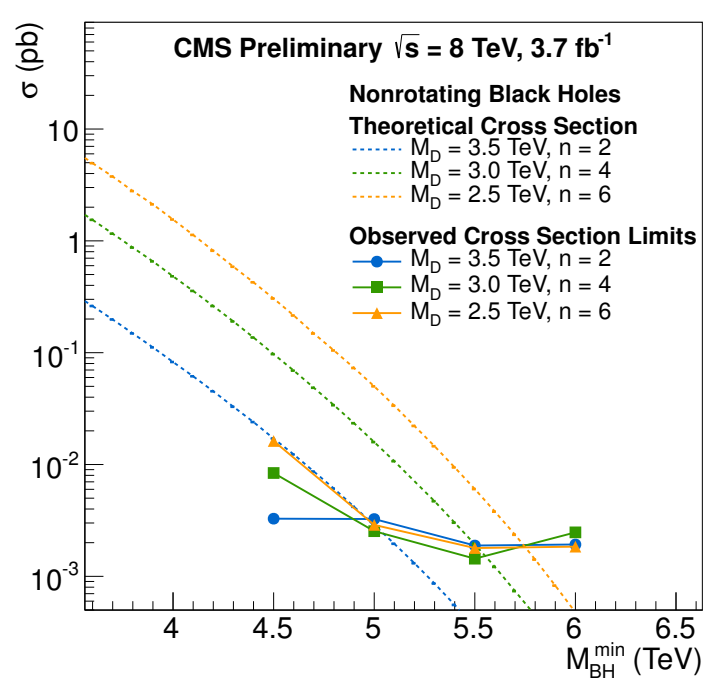

(a) model-dependent cross section limits

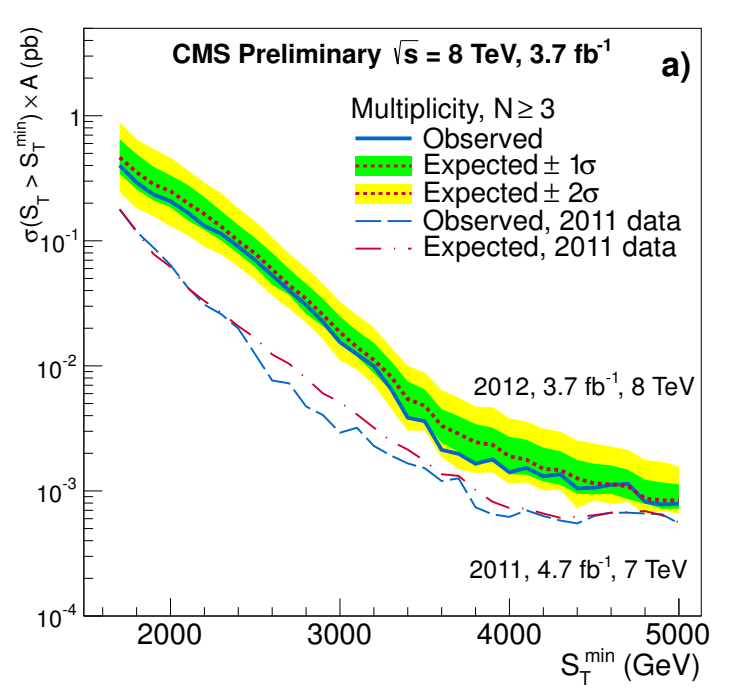

(b) model-independent limits

Figure 12: Experimental (solid lines) cross section limits for various black hole parameter sets compared with the theoretical signal production cross sections (dotted lines, left plot.) Model-independent 95\% confidence level upper limits on a signal cross section times acceptance for $\mathrm{ST}>\mathrm{ST}_{\min }$ as a function of STmin for $\mathrm{N} \geq 3$. The blue (red) lines correspond to an observed (expected) limit (right), [34].

\section{Summary: exotics}

The list of results of searches for exotic physics has grown long, as is seen in the bar plot Figure 13. No statistically significant excess beyond the standard model predictions has been found in the entire physics program. 

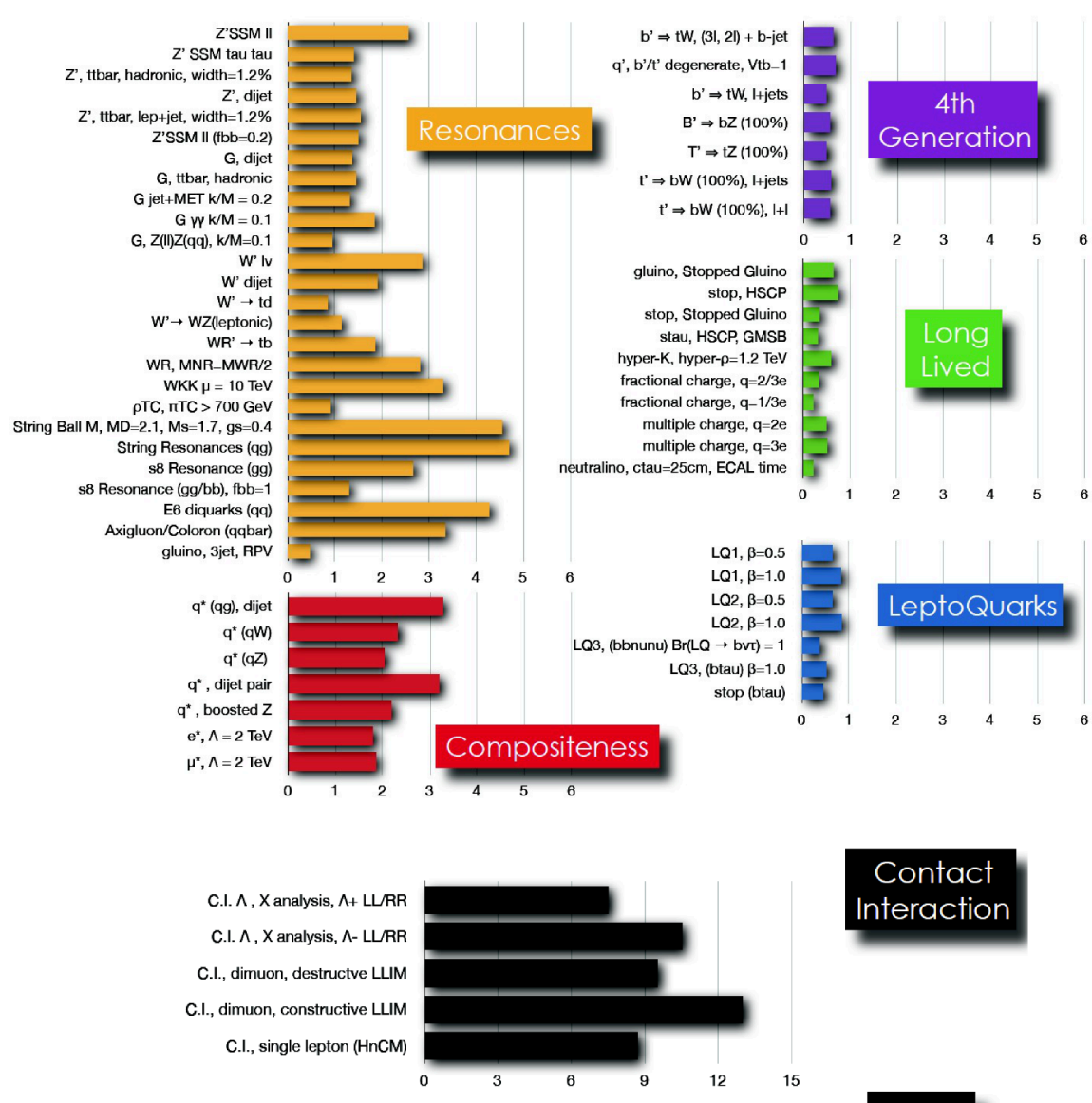

\section{Contact}
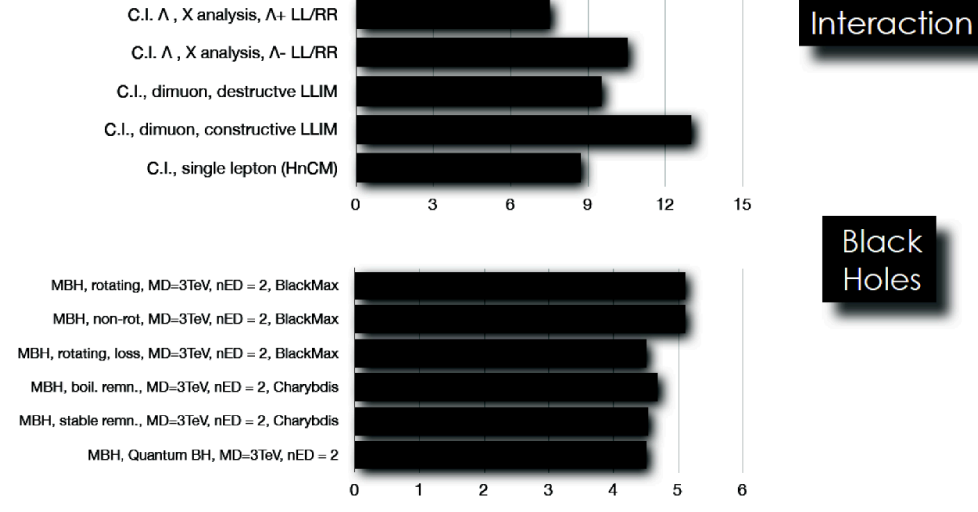

Figure 13: Summary: searches for exotic physics

\section{Summary}

A wide variety of searches for physics beyond the standard model have been performed, no significant excess is to be reported. Limits are set in a large array of models. The Standard Model continues to be a success story, despite all our efforts. The analyses of the full $8 \mathrm{TeV}$ datasets are in progress and will continue to search for physics beyond the standard model. A final word on the hierarchy problem has not been spoken, yet: it will take the results of the $8 \mathrm{TeV}$ dataset and quite possibly also of the future $13 \mathrm{TeV}$ datasets to reach to more conclusive answers.

\section{References}

[1] CMS Collaboration Collaboration, S. Chatrchyan et al., "Observation of a new boson at a mass of 
$125 \mathrm{GeV}$ with the CMS experiment at the LHC," Phys.Lett. B716 (2012) 30-61, 1207.7235.

[2] ATLAS Collaboration Collaboration, G. Aad et al., "Observation of a new particle in the search for the Standard Model Higgs boson with the ATLAS detector at the LHC," Phys.Lett. B716 (2012) 1-29, 1207.7214.

[3] J. Wess and B. Zumino, "Supergauge Transformations in Four-Dimensions," Nucl. Phys. B 70 (1974) 39-50.

[4] Y. Golfand and E. Likhtman, "Extension of the Algebra of Poincare Group Generators and Violation of p Invariance," JETP Lett. 13 (1971) 323-326.

[5] A. Neveu and J. Schwarz, "Quark Model of Dual Pions," Phys.Rev. D4 (1971) 1109-1111.

[6] P. Ramond, “Dual Theory for Free Fermions," Phys.Rev. D3 (1971) 2415-2418.

[7] D. Volkov and V. Akulov, "Is the Neutrino a Goldstone Particle?," Phys.Lett. B46 (1973) 109-110.

[8] G. R. Farrar and P. Fayet, "Phenomenology of the Production, Decay, and Detection of New Hadronic States Associated with Supersymmetry," Phys.Lett. B76 (1978) 575-579.

[9] A. H. Chamseddine, R. Arnowitt, and P. Nath, "Locally supersymmetric grand unification," Phys. Rev. Lett. 49 (1982) 970.

[10] R. Arnowitt and P. Nath, "Supersymmetric mass spectrum in SU(5) supergravity grand unification," Phys. Rev. Lett. 69 (1992) 725.

[11] G. L. Kane, C. F. Kolda, L. Roszkowski, and J. D. Wells, "Study of constrained minimal supersymmetry," Phys. Rev. D 49 (1994) 6173, hep-ph/ 9312272.

[12] MSSM Working Group Collaboration, A. Djouadi et al., "The Minimal supersymmetric standard model: Group summary report," hep-ph/9901246.

[13] A. Djouadi, J.-L. Kneur, and G. Moultaka, "SuSpect: A Fortran code for the supersymmetric and Higgs particle spectrum in the MSSM," Comput.Phys.Commun. 176 (2007) 426-455, hep-ph/0211331.

[14] C. F. Berger, J. S. Gainer, J. L. Hewett, and T. G. Rizzo, “Supersymmetry Without Prejudice,” JHEP 0902 (2009) 023, 0812.0980.

[15] M. Cahill-Rowley, J. Hewett, A. Ismail, and T. Rizzo, "SUSY Without Prejudice at the 7 and $8 \mathrm{TeV}$ LHC: Gravitino LSPs,” 1211.7106.

[16] B. Knuteson and S. Mrenna, "BARD: Interpreting new frontier energy collider physics," hep-ph/0602101.

[17] N. Arkani-Hamed, B. Knuteson, S. Mrenna, P. Schuster, J. Thaler, N. Toro, and L.-T. Wang, "MARMOSET: The Path from LHC Data to the New Standard Model via On-Shell Effective Theories." 2007.

[18] LHC New Physics Working Group Collaboration, D. Alves et al., "Simplified models for LHC new physics searches," J. Phys. G 39 (2012) 105005, 1105.2838.

[19] CMS Collaboration, "Interpretation of searches for supersymmetry with simplified models," 1301.2175.

[20] "Search for supersymmetry with taus using the razor variables," Tech. Rep. CMS-PAS-SUS-11-029, CERN, Geneva, 2012. 
[21] "Scalar Top Quark Search with Jets and Missing Momentum in pp Collisions at sqrt(s) = 7 TeV," Tech. Rep. CMS-PAS-SUS-11-030, CERN, Geneva, 2012.

[22] "Search for direct EWK production of SUSY particles in multilepton modes with 8 TeV data," Tech. Rep. CMS-PAS-SUS-12-022, CERN, Geneva, 2012.

[23] "Search for direct top squark pair production in events with a single isolated lepton, jets and missing transverse energy at sqrt(s) = $8 \mathrm{TeV}$, , Tech. Rep. CMS-PAS-SUS-12-023, CERN, Geneva, 2012.

[24] "A search for anomalous production of events with three or more leptons using 9.2 $\mathrm{fb}^{-1}$," Tech. Rep. CMS-PAS-SUS-12-026, CERN, Geneva, 2012.

[25] "Search for RPV supersymmetry with three or more leptons and b-tags," Tech. Rep. CMS-PAS-SUS-12-027, CERN, Geneva, 2012.

[26] "Search for supersymmetry in final states with missing transverse energy and 0, 1, 2, 3, or at least 4 b-quark jets in $8 \mathrm{TeV}$ pp collisions using the variable $\alpha_{T}$," Tech. Rep. CMS-PAS-SUS-12-028, CERN, Geneva, 2012.

[27] "Search for supersymmetry in events with same-sign dileptons and b-tagged jets with $8 \mathrm{TeV}$ data," Tech. Rep. CMS-PAS-SUS-12-029, CERN, Geneva, 2012.

[28] R. Barbieri and G. Giudice, “Upper Bounds on Supersymmetric Particle Masses,” Nucl.Phys. B306 (1988) 63.

[29] S. Dimopoulos and G. Giudice, "Naturalness constraints in supersymmetric theories with nonuniversal soft terms,” Phys.Lett. B357 (1995) 573-578, hep-ph/ 9507282.

[30] R. Barbieri and D. Pappadopulo, "S-particles at their naturalness limits," JHEP 0910 (2009) 061, 0906.4546.

[31] M. Papucci, J. T. Ruderman, and A. Weiler, "Natural SUSY Endures,” JHEP 1209 (2012) 035, 1110.6926.

[32] CMS Collaboration Collaboration, S. Chatrchyan et al., "Search for new physics in final states with a lepton and missing transverse energy in pp collisions at the LHC," 1302.2812.

[33] G. Altarelli, B. Mele, and M. Ruiz-Altaba, "Searching for new heavy vector bosons in p anti-p colliders," Z.Phys. C45 (1989) 109.

[34] CMS Collaboration Collaboration, "Search for Black Holes in pp Collisions at sqrt(s) = 8 TeV,".

[35] R. M. Harris and K. Kousouris, "Searches for Dijet Resonances at Hadron Colliders," Int.J.Mod.Phys. A26 (2011) 5005-5055, 1110 . 5302.

[36] CMS Collaboration Collaboration, S. Chatrchyan et al., "Search for narrow resonances using the dijet mass spectrum in pp collisions at $\operatorname{sqrt}(\mathrm{s})=8 \mathrm{TeV}, " 1302.4794$. 